\title{
Grammaire et enseignement du français, 1500-1700.
}

Édité par Jean De Clercq, Nico Lioce, Pierre Swiggers. Orbis supplementa, tome 16. Leuven - Paris - Sterling (Virginia) : Peeters, 2000, 671 p.

\section{Henri Besse}

\section{(2) OpenEdition}

\section{Journals}

Édition électronique

URL : https://journals.openedition.org/dhfles/1277

DOI : $10.4000 /$ dhfles. 1277

ISSN : 2221-4038

Éditeur

Société Internationale pour l'Histoire du Français Langue Étrangère ou Seconde

Édition imprimée

Date de publication : 1 juin 2004

Pagination : 168-173

ISSN : 0992-7654

\section{Référence électronique}

Henri Besse, "Grammaire et enseignement du français, 1500-1700. ", Documents pour l'histoire du français langue étrangère ou seconde [En ligne], 32 | 2004, mis en ligne le 01 janvier 2012, consulté le 27 mai 2021. URL : http://journals.openedition.org/dhfles/1277 ; DOI : https://doi.org/10.4000/dhfles. 1277

Ce document a été généré automatiquement le 27 mai 2021.

(c) SIHFLES 


\section{Grammaire et enseignement du français, 1500-1700.}

Édité par Jean De Clercq, Nico Lioce, Pierre Swiggers. Orbis supplementa, tome 16. Leuven - Paris - Sterling (Virginia) : Peeters, 2000, 671 p.

\section{Henri Besse}

1 Ce gros volume regroupe trente contributions - y comprise celle, dont le titre précise qu'il s'agit de la « Grammaire et (de l')enseignement du français langue étrangère entre 1500 et 1700 », où ses trois « éditeurs » résument magistralement les vingt-neuf autres -, dont cinq en anglais et cinq en allemand. Un index des matières, parfois lacunaire (il y est question de «routine » en d'autres endroits que p. 97-99), et un index des noms, plus complet (incluant les auteurs des bibliographies propres à chaque contribution), en font un ouvrage indispensable à quiconque s'intéresse à l'histoire de la grammaire appliquée à la langue française. Sa table des matières distingue deux parties, la première étant réservée aux « questions de méthodologie et aspects institutionnels », la seconde distribuant les études selon l'aire géographique concernée (Scandinavie, Angleterre et Écosse, Pays-Bas, région rhénane, péninsule Ibérique, Italie). Ce qui semble un peu arbitraire, certains textes de la première partie étant parfois aussi localisés que ceux de la seconde, et certains de la seconde aussi méthodologiques et institutionnels que ceux de la première. Les lecteurs de Documents y retrouveront les noms de nombreux membres de la SIHFLES, société dont les trois « éditeurs » affirment qu'elle «se consacre exclusivement à la didactique du FLE» (p. IX, note 1) essentiellement serait plus exact.

2 L'ouvrage n'en constitue pas moins un précieux outil à même de compléter, pour s'en tenir à cette seule publication qui n'y est pas citée (sauf inadvertance de notre part), le Corpus représentatif des grammaires et des traditions linguistiques, publié par la revue Histoire Épistémologie Langage. Le tome 1 de ce Corpus (H. E. L., Hors-Série $n^{\circ} 2,1998$ ) répertorie, pour les $\mathrm{XVI}^{\mathrm{e}}$ et $\mathrm{XVII}{ }^{\mathrm{e}}$ siècles, vingt-deux "Grammaires françaises et grammaires générales d'expression française ». Par "grammaires françaises", on entend en général des manuels destinés à l'enseignement, médiatisés ou non par un enseignant, des « règles » de la langue française, plus précisément d'une de ses variétés 
(dite normée ou standard). Soit leurs auteurs présupposent que cette langue est déjà parlée, au moins dans une de ses variétés, par les utilisateurs potentiels de son manuel, et l'on a affaire, selon le jargon actuel, à une « grammaire du français langue maternelle (FLM) ». Soit leurs auteurs présupposent que cette langue n'est pas encore comprise et parlée, dans l'une ou l'autre de ses variétés, par une partie au moins des utilisateurs potentiels (ceux qui l'utiliseront pour l'apprendre et non pour l'enseigner), et l'on a affaire, toujours selon le jargon actuel, à une " grammaire du français langue étrangère (FLE) ». Pour le seul XVI ${ }^{e}$ siècle, dix " grammaires françaises » - nous en écartons deux manuels qui sont en fait des "grammaires latines " écrites ou traduites en français, sans doute parce que le latin commençait à être perçu en France comme un peu «étranger " - sont l'objet d'une notice dans le Corpus : celle de J. Palsgrave (1530), rédigée en anglais ; celle de J. Dubois dit Sylvius (1531), rédigée en latin, «la première grammaire du français écrite en France par un Français ${ }^{1} »$; celle de L. Meigret (1550), écrite en français par un Lyonnais (comme le précise son titre); celle de J. Pillot (1550) dit Pillotus, rédigée en latin; celle de R. Estienne (1557), rédigée d'abord en français mais vite traduite en latin (1558); celle de J. Garnier (1558), rédigée en latin ; celles de P. de la Ramée dit Ramus, l'une publiée en 1562 et relativement courte, l'autre en 1572 et plus développée, toutes deux en français; celle d'A. Cauchie dit Caucius (1570), rédigée en latin et en allemand; et celle de J. Bosquet (1586, mais dont la 1 ère édition est sans doute antérieure) rédigée en français. Notre ouvrage se réfère souvent à ces dix "grammaires françaises", de Palsgrave (cité 29 fois dans l'index des noms) à Cauchie (cité 4 fois), sans qu'elles y soient pour autant l'objet d'études spécifiques. On notera que les informations qu'on y trouve à leur propos ne coïncident pas toujours avec celles du Corpus: par exemple, pour Pillot, ni le titre de la $1^{{ }^{\text {ère }}}$ édition ni la liste de ses republications ne sont exactement les mêmes (voir p. 426, note 7) - ce qui devrait stimuler de nouvelles investigations. Mais nombre d'autres « grammaires françaises » $y$ apparaissent, dont (pour ne citer que celles du XVI ${ }^{e}$ siècle qui y font l'objet d'analyses un peu développées) deux de G. Meurier (1557 et 1563), rédigées essentiellement en français mais destinées à un public anglais, allemand ou flamand; celle de B. de Sotomayor (1565) rédigée en espagnol et qui semble en partie plagiée de Meurier (1557) ; deux de G. Du Vivier, celle de (1566) « mise en François \& Allemang ", et celle de (1568) « expliquee en Aleman»; celle de P. Heyns (1571) écrite en flamand; ou encore celles de J. Serreius (1598 et 1600), rédigées en latin. Autant de "grammaires françaises » qui sont au moins aussi « représentatives ", à s'en tenir à leur diffusion au cours des $\mathrm{XVI}^{e}$ et XVII ${ }^{e}$ siècles, que celles répertoriées par le Corpus (deux éditions pour le manuel de Palsgrave ; une seule pour le Sylvius et pour le Meigret). On savait que les trois premières "grammaires françaises» (si l'on ajoute celle de Barton à celles de Palsgrave et Dubois) étaient des "grammaires du FLE »; notre ouvrage confirme que le français a commencé à être étudié grammaticalement plus comme FLE que comme FLM.

Mais la distinction " grammaire du FLE vs FLM ", qui ne fut guère conceptualisée avant la seconde moitié $\mathrm{du} \mathrm{XX}^{\mathrm{e}}$ siècle, n'est pas toujours évidente. Si une "grammaire française" est "bilingue», au sens que le français analysé (que le corpus appelle «langue-cible») l'est au moyen d'une autre langue (que le Corpus appelle "métalangue »), laquelle est présupposée connue de ses utilisateurs potentiels (parce qu'il s'agit de leur L1 ou d'une première L2, tel le latin), il est clair qu'on a affaire à une "grammaire du FLE». Mais si la "grammaire» est "monolingue», si le français analysé l'est au moyen de lui-même, il est plus difficile de savoir si l'on a affaire à une "grammaire du FLM" ou à une "grammaire du FLE». Il faut alors s'appuyer sur 
d'autres critères moins évidents (ce qu'en dit l'auteur, la manière dont il a élaboré son manuel...) : la "grammaire française » de Ch. Maupas (1607), tout aussi «monolingue " que celles de Meigret ou d'Estienne (1557), est d'abord une " grammaire du FLE », parce que son auteur précise, dans son titre même, l'avoir conçue « en faveur des estrangiers qui [...] sont désireux » de "nostre langue », et qu'elle correspond à son expérience d'enseignement du FLE à Blois (où ses élèves pouvaient être de diverses nationalités et ne pas tous connaître le latin). Et la distinction se brouille encore davantage quand la «métalangue » d'un manuel "monolingue» est traduite dans une autre langue (tel Estienne traduisant en latin, dès 1558, sa "grammaire française » de 1557), ou quand un manuel «bilingue» est traduit (exemples en «langue-cible» compris) dans une troisième, quatrième, cinquième... langue, engendrant ainsi, à moindre coût (y compris intellectuel), des "grammaires multilingues ».

Il n'en reste pas moins qu'à ne pas prendre en compte cette distinction, on risque anachronismes et approximations. Par exemple, dans une "grammaire du FLM", l'orthographe l'emporte en général sur la prononciation, simplement parce que ses utilisateurs sont censés déjà parler le français ; dans une "grammaire du FLE », quand il n'y avait pas de moyens pour enregistrer la parole, c'est l'oralisation des textes en L2 qui importait. Sur ce point, Grammaire et enseignement du français nous paraît clarifier nombre d'études antérieures (où il était question, non sans anachronisme, de phonétique voire même de phonologie) en parlant (après D. A. Kibbee, voir Documents $\mathrm{n}^{\circ} 3$, juin 1989) d'une "démarche grapho-phonétique" ( $\mathrm{p}$. XIII), qui consiste à enseigner «le phonétisme du français comme s'il était le résultat de la correcte oralisation des textes écrits lus à haute voix» (p. 62, note 2). Ce qui conduit, en particulier, à distinguer soigneusement les « diphtongues » ou les «triphtongues » des grammairiens anciens, qui n'étaient le plus souvent que des digrammes ou trigrammes, de ce que les phonéticiens ou les phonologues entendent par là. On peut regretter que nombre de contributions n'aient pas apporté le même soin à clarifier en quoi l'enseignement de la morphologie et de la syntaxe dans une « grammaire du FLE » ne peut pas être le même que celui d'une "grammaire du FLM ». En particulier, quand il s'agit de débutants non lettrés, tels ces marchands ou ces femmes n'ayant pas été familiarisés antérieurement, par l'étude scolaire du latin, à un certain nombre d'outils métalinguistiques (telles les catégories du mot ou des parties du discours). On trouve certes, ça et là, des remarques fort pertinentes, sur la catégorie de l'article qui était peu utilisée dans les "grammaires latines"; sur la nécessité ou non de donner une définition de ces outils métalinguistiques sans lesquels on ne peut réfléchir grammaticalement, selon que les élèves les ont déjà appris ou non, selon qu'il s'agit d'un manuel destiné à l'autodidaxie ou non ; sur les paradigmes des exemples illustrant une règle en place de son énoncé explicite; ou sur une analyse différentielle ou contrastive entre le français et la langue maternelle des élèves. Mais certaines contributions nous paraissent critiquer les « grammaires françaises » qu'elles prennent pour objet sur le seul mode du "manque ", en se référant à quelque modèle idéal (de rationalité, d'exhaustivité, de progrès...), comme si ces « grammaires » étaient d'abord destinées à de futurs grammairiens ou linguistes. Posture évaluative qui nous paraît rejoindre celle de ce rédacteur du Corpus pour qui la « grammaire » de Meigret est une "grammaire complète", et celle d'Estienne (nous soulignons), une "grammaire complète, mais pédagogique (nombreux tableaux) ».

5 M. Colombo Timelli (p. 570) dénonce, à juste titre nous semble-t-il, « une nuance de mépris» dans le fait de qualifier (au sens quasi juridique du terme) certaines 
«grammaires » de «pratiques » ou de "pédagogiques», alors que d'autres sont, par "appréciation positive implicite», qualifiées de "théoriques", "savantes", voire "scientifiques». Qualifications qui, sous ces termes ou d'autres, nous semblent trop souvent présentes dans notre ouvrage. Que certaines " grammaires françaises » soient, pour un même projet didactique, mieux faites que d'autres est une évidence. Mais existe-t-il une "grammaire française " du XVI ${ }^{e}$ ou XVII ${ }^{e}$ siècles qui ne soit pas, à un titre ou à un autre, quelque peu "pratique » ou "pédagogique »? On peut en douter, même pour la Grammaire générale et raisonnée de $\mathrm{Cl}$. Lancelot et $\mathrm{A}$. Arnauld. Si elle est "d'expression française ", comme il est dit dans le Corpus, c'est aussi parce que, dans les Petites écoles de Port-Royal, on y commençait par l'apprentissage non du latin mais du FLM ; et si Lancelot, dans sa Préface, dit qu'il s'agit « de faire par science ce que les autres font seulement par coutume ", c'est sans doute aussi parce qu'il y voit, en tant qu'auteur de "grammaires latine, grecque, italienne et espagnole", un moyen de faciliter l'apprentissage ultérieur " par règles » d'autres langues que le FLM. Il faudra attendre les 1500 pages de N. Beauzée (1767) pour avoir une "grammaire générale " dont on peut dire, sans courir le risque d'être démenti, qu'elle n'est ni " pratique » ni " pédagogique » (elle ne connaîtra d'ailleurs qu'une seule édition au XVIII ${ }^{\mathrm{e}}$ siècle et une seule réédition au XIX $\mathrm{X}^{\mathrm{e}}$ siècle).

Une "grammaire du FLM" se distingue aussi, du XVI ${ }^{e}$ au XVIII ${ }^{e}$ siècles, d'une "grammaire du FLE», par le fait que celle-ci inclut souvent, dans la tradition des " manières de langage ", " glossaires » ou " colloques » du Moyen Age, des dictionnaires bilingues (voire multilingues), des dialogues familiers (en français, traduits ou non dans une autre langue), des listes de proverbes, des anecdotes... C'est que si, dans l'enseignement d'une "grammaire du FLM », le "par règles " peut se passer du "par usage ", puisque les élèves maîtrisent déjà un certain usage du français, il n'en va pas de même dans l'enseignement d'une "grammaire du FLE ", où le " par règles » ne peut se passer du "par usage ", qu'il leur soit inculqué avant ou après celles-ci. Le Corpus écarte le plus souvent ce type de manuels, et quand il en retient un, la partie "par usage » y est totalement ignorée. Ainsi, pour Palsgrave, il n'y est question (cf. rubrique 10, qui est supposée donner une "description condensée du contenu ») que du fyrst boke et du seconde boke; on n'y trouve rien sur The thirde boke, qui contient certaynes tables contayning all the wordes in our tong [l'anglais] after the order of a, b, c, with the frenche wordes joyned unto them. Grammaire et enseignement $d u$ français ne dédaigne pas les études portant sur ce "par usage ", même si l'on peut parfois regretter qu'elles se bornent à une simple description sans trop pousser l'analyse (en ayant, par exemple, recours aux "outils» de l'analyse de discours), et sans trop s'interroger sur la compatibilité du " par usage » avec le « par règles ».

7 M. Colombo Timelli cite en exergue de sa contribution le Aliud est grammaticè, aliud Gallicè loqui de Ph. Neretti (1697), déjà cité par N. Minerva en 1991 (Documents n 8, p. 16) ; mais Neretti ne faisait - après et avant bien d'autres - qu'appliquer au français ce que Quintilien avait déjà dit du latin : aliud esse Latine, aliud grammatica loqui. On a fait remarquer que si l'histoire a invalidé la plus grande partie de la physique d'Aristote, elle a épargné la plus grande partie de sa métaphysique. On a parfois l'impression qu'il en va de même pour la grammaire et l'enseignement des langues. 


\section{NOTES}

1. Ce qui pose la question de savoir ce qu'on entendait alors par «France " et par «un Français » : Pillot était de Lorraine, dont François $1^{\text {er }}$ et Charles Quint avaient reconnu en juin 1536 la neutralité.

\section{AUTEUR}

\section{HENRI BESSE}

ENS Lettres \& Sciences humaines - UMR CNRS 7597 